\title{
Ixeris dentata extract regulates salivary secretion through the activation of aquaporin-5 and prevents diabetes-induced xerostomia
}

This article was published in the following Dove Press journal:

Journal of Experimental Pharmacology

31 July 2017

Number of times this article has been viewed

\author{
Kashi Raj Bhattarai' \\ Sang-Won Lee ${ }^{2}$ \\ Seung Hyun Kim ${ }^{3}$ \\ Hyung-Ryong $\mathrm{Kim}^{4}$ \\ Han-Jung Chae'
}

'Department of Pharmacology and Institute of New Drug Development, School of Medicine, Chonbuk National University, Jeonju, ${ }^{2}$ Department of Herb Crop Resources, National Institute of Horticultural and Herbal Science, Rural Development Administration, Eumseong-gun, ${ }^{3}$ College of Pharmacy, Yonsei Institute of Pharmaceutical Science, Yonsei University, Incheon, ${ }^{4}$ Graduate School, Daegu Gyeongbuk Institute of Science and Technology, Daegu, Republic of Korea
Correspondence: Han-Jung Chae Department of Pharmacology and Institute of New Drug Development, School of Medicine, Chonbuk National University, Jeonju, Republic of Korea

$\mathrm{Tel}+82632703092$

Fax +82632752855

Email hjchae@jbnu.ac.kr

Hyung-Ryong Kim Graduate School, DGIST, Daegu, 42988, Republic of Korea

$\mathrm{Tel}+82638506640$

Fax +82 638540285

Email hrkim@dgist.ac.kr

\begin{abstract}
The aim of this study was to investigate the effects of Ixeris dentata (IXD) extract to improve the salivation rate in dry mouth induced by diabetes. Both control and diabetic rats were treated with a sublingual spray of either water or IXD extract to determine the effects of IXD on salivation. During the study, we observed that IXD extract treatment increased the salivary flow rate in diabetic rats. The expression of $\alpha$-amylase was increased significantly in both saliva and glandular tissue lysates of IXD-treated diabetic rats. Aquaporin-5 protein expression was abnormally low in the salivary glands of diabetic rats, which increased hyposalivation and led to salivary dysfunction. However, a single oral spray of IXD extract drastically increased the expression of aquaporin-5 in salivary gland acinar and ductal cells in diabetic rats. Moreover, IXD extract induced expression of $\mathrm{Na}^{+} / \mathrm{H}^{+}$exchangers in the salivary gland, which suggests that $\mathrm{Na}^{+} / \mathrm{H}^{+}$exchangers modulate salivary secretions and aid in the fluid-secretion mechanism. Furthermore, transient treatment with IXD extract increased the intracellular calcium in human salivary gland cells. Taken together, these results suggest the potential value of an IXD extract for the treatment of diabetes-induced hyposalivation and xerostomia.
\end{abstract}

Keywords: Ixeris dentata, hyposalivation, amylase, aquaporin-5, xerostomia, diabetes

\section{Introduction}

Ixeris dentata (IXD, Asteraceae) is a traditional herbal medicinal plant consumed mainly in Korea, Japan and China for the treatment of hepatitis, indigestion, diabetes, allergies and tumors. ${ }^{1,2}$ The methanolic extract of IXD has been shown to reduce triglyceride and cholesterol levels in streptozotocin (STZ)-induced diabetic rats. ${ }^{3}$ The antioxidant properties of IXD have been found to reduce oxidative stress by maintaining glutathione concentrations in the brains of mice. ${ }^{4}$ The whole-saliva flow rate and water secretion are abnormally low in diabetic patients who complain of xerostomia. ${ }^{5}$ In patients with oral dryness, the loss of the protective effects of salivary buffers, proteins and mucins accelerates the destruction of salivary gland tissues. ${ }^{6}$

Saliva is composed of $>99 \%$ water and $<1 \%$ solids, mostly proteins and salts. ${ }^{7}$ Hyposalivation and chronic xerostomia increase alterations in speech, difficulty chewing and swallowing, burning sensations in the mouth, dental caries and the risk of oral infections. ${ }^{8} \alpha$-Amylase, a major digestive enzyme found in saliva, is responsible for the initial digestion of starch and is a good indicator of proper functioning salivary glands. ${ }^{9}$ It is synthesized in the acinar cells of the salivary glands and stored in secretory granules in these cells. Aquaporin-5 (AQP5) is the main channel protein expressed on the apical membrane of salivary acinar cells, and it plays a prominent role in saliva secretion. ${ }^{10}$ Abnormal expression of AQP5 has been observed in autoimmune 
diseases, such as Sjogren's syndrome, ${ }^{11}$ and in non-obese diabetic mice. ${ }^{12}$

In the present study, we have chosen an oral spray delivered via the sublingual route for drug administration since the sublingual area is more highly permeable than other areas ${ }^{13}$ and is useful for observing the rapid onset of action of the drug. Oral sprays have been used effectively in various conditions and diseases such as angina and nausea. ${ }^{14}$ This in vivo study shows that treatment with an oral spray of IXD extract increases salivary secretion through activation of the water channel protein AQP5 to ameliorate xerostomia.

\section{Materials and methods}

\section{Plant material and preparation}

The roots of IXD were harvested in Dangin, Republic of Korea, in March 2014. Voucher specimens (ID 2014-01) were identified by the National Institute of Horticultural and Herbal Science at the Rural Development Administration of Korea and deposited at the College of Pharmacy of Yonsei University in Incheon, Korea. The dried roots of IXD were cut into smaller pieces and further ground into powder. The powder $(1.2 \mathrm{~kg})$ was extracted 3 times with $3 \mathrm{~L}$ of ethanol in an ultrasonic apparatus for $3 \mathrm{~h}$ at $35^{\circ} \mathrm{C}$. Removal of the solvent in vacuo yielded $30.5 \mathrm{~g}$ (2.5\% dry weight) of ethanol extract. The ethanol extract was suspended in water to the desired concentration before use.

\section{Chemicals and reagents}

Chemicals, including streptozotocin (STZ), citric acid, pilocarpine hydrochloride, and 1, 2-Bis (2-aminophenoxy) ethane-N, N, N', N'- tetraacetic acid tetrakis (acetoxymethyl ester) (BAPTA-AM) were obtained from Sigma-Aldrich Co. (St. Louis, MO, USA).

\section{Animals}

Forty Sprague-Dawley rats (7 weeks old, male, 200-230 g) were obtained from Damul Science (Daejeon, Republic of
Korea), divided into 4 groups of 10 animals per group, and kept under standard living conditions $\left(22^{\circ} \mathrm{C} \pm 2{ }^{\circ} \mathrm{C}, 55 \%-60 \%\right.$ relative humidity, and a $12-\mathrm{h}$ light/dark cycle). The rats were acclimatized to our laboratory conditions for 1 week before use in the experiments. A diet of tap water and standard food pellets was available ad libitum throughout the experimental period. All animal care procedures and experiments conformed to the institutional guidelines of Chonbuk National University. All the animal use protocols were approved by the Institutional Animal Care and Use Committee (IACUC, CBNU 2017-0014) of Chonbuk National University Laboratory Animal Center.

\section{Experimental design}

A schematic of the experimental procedure is given in Figure 1. Briefly, 2 weeks after either vehicle or STZ injection, the animals were anesthetized prior to sublingual treatment. The normal control group was sprayed with water (control + water) under the tongue $(1$ spray $=50 \mu \mathrm{L})$. The second normal group was sublingually treated with an IXD extract (control+IXD, $10 \mathrm{mg} / \mathrm{kg}$ ). The diabetic control rats were sprayed with water (STZ + water). The diabetic treated rats were sprayed with an IXD extract (STZ+IXD, $10 \mathrm{mg} / \mathrm{kg}$ ). Saliva was collected and then the animals were sacrificed. After sacrificing, the bilateral submandibular glands (SMGs) were carefully excised and weighed. One lobe was immediately frozen, and the remaining section was kept in formalin (formaldehyde 3.7\%; Dana Korea, Seoul, Republic of Korea) for histological examinations.

\section{Induction of diabetes}

Type 1 diabetes was induced by a single intraperitoneal injection of freshly prepared STZ $(65 \mathrm{mg} / \mathrm{kg})$ dissolved in cold $0.1 \mathrm{M}$ citrate buffer, $\mathrm{pH} 4.5$. Citrate buffer alone was injected in control rats as a vehicle. Basal blood glucose levels were tested 3 days after injection using the tail vein puncture method in overnight-fasted control and diabetic

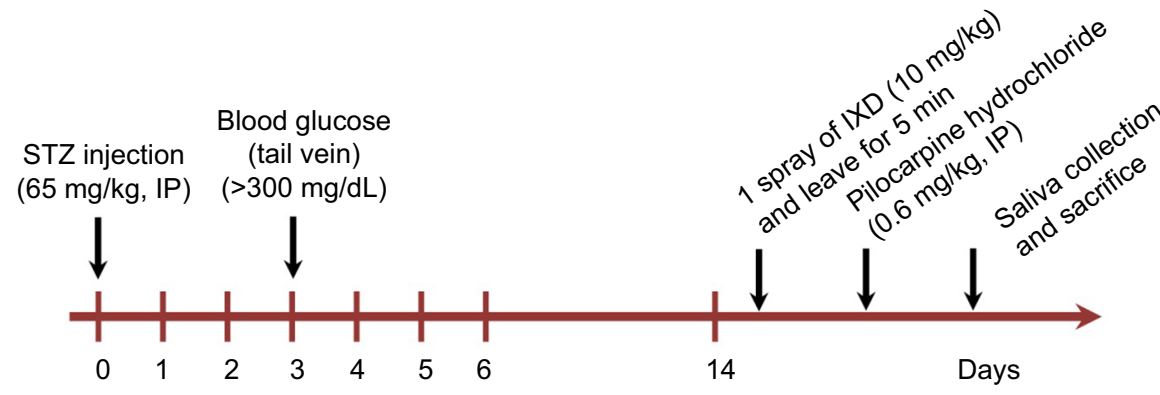

Figure I Schematic diagram of the experimental design.

Abbreviations: IP, intraperitoneal, IXD, Ixeris dentata; STZ, streptozotocin. 
rats. The systemic glucose concentration was assessed using a glucometer (Accu-Chek, Roche, Germany). Blood glucose concentrations higher than $300 \mathrm{mg} / \mathrm{dL}$ were considered to be diabetic and those animals were used in the diabetic model. Glucometer readings of "Hi" were considered to be $600 \mathrm{mg} / \mathrm{dL}$ as described previously. ${ }^{15}$

\section{Collection of total saliva}

Two weeks after either vehicle or STZ injection, a single spray of water or IXD extract $(10 \mathrm{mg} / \mathrm{kg})$ was given beneath the tongue of anesthetized rats and left for $5 \mathrm{~min}$. Pilocarpine $(0.6 \mathrm{mg} / \mathrm{kg})$ was injected intraperitoneally and saliva was collected using pre-weighed, small cotton balls (as described previously ${ }^{16}$ ) for up to $30 \mathrm{~min}$. The total saliva collected was calculated as the difference of the cotton balls' weight pre- and post-collection, which was then converted from grams to milliliters. The salivary flow rate was calculated as saliva amount (in microliters) divided by collection time (in minutes).

\section{Salivary total protein concentration measurement}

Immediately after saliva collection, the protein concentration in the saliva was determined using the Bradford method (Bradford assay; BioRad, Hercules, CA, USA).

\section{Immunoblotting}

Western blotting was performed using SMG tissue homogenates and whole saliva. The homogenate was prepared by homogenizing the SMG tissue in radioimmunoprecipitation assay lysis buffer. The composition of lysis buffer was prepared as described in Lee et al. ${ }^{17}$ Total protein was quantified using the BioRad Protein assay (BioRad). Thirty $\mu \mathrm{g}$ of total protein were loaded per lane, separated by sodium dodecyl sulfate polyacrylamide gel electrophoresis (SDS-PAGE; BioRad), and transferred to a polyvinylidene fluoride membrane. The membrane was blocked with $5 \%$ skim milk in tris-buffered saline $(0.137 \mathrm{M} \mathrm{NaCl}, 0.025 \mathrm{M}$ tris, $\mathrm{pH} 7.4)$ containing $0.1 \%$ Tween 20 (TBST). The primary antibodies for amylase and AQP5 (Santa Cruz Biotechnology Inc., Dallas, TX, USA) were diluted with 5\% skim milk at a ratio of 1:1000 and incubated with the membrane overnight at $4^{\circ} \mathrm{C}$. The secondary antibodies (Santa Cruz Biotechnology Inc.) (anti-mouse for amylase and anti-goat for AQP5) were diluted with 5\% skim milk at a ratio of 1:5000 and incubated for $1 \mathrm{~h}$ at room temperature. The proteins were visualized with enhanced chemiluminescence (ECL) reagents (SuperDetect $^{\mathrm{TM}}$ ECL Western Blotting Detection Reagent; Dae
Myung Science Co., Ltd., Seoul, Korea). The membranes were exposed to imaging film (Kodak BioFlex; Econo Scientific Supplies, Citrus Heights, CA, USA) and developed using a Kodak X-OMAT 1000A processor (Kodak, Toronto, ON, Canada). For Coomassie Brilliant Blue R-250 staining (CBB, Figure $3 \mathrm{~B}$ ), equal volumes of saliva samples were used to observe the total protein expression in saliva. The protein was separated using SDS-PAGE, stained with CBB (Bio-Rad) and de-stained using a $30 \%$ methanol $/ 10 \%$ acetic acid solution.

\section{Hematoxylin and eosin staining}

Routinely processed paraffin-embedded SMG tissue was sliced to a thickness of $5 \mu \mathrm{m}$. The sections were deparaffinized and rehydrated in decreasing concentrations of alcohol. They were then washed in tap water and stained with Harris hematoxylin and $1 \%$ eosin. The sections were then dehydrated using increasing concentrations of alcohol and cleaned with xylene. Finally, the sections were mounted using anhydrous mounting media (Merck, Darmstadt, Germany) and observed with a light microscope.

\section{Immunohistochemistry}

Immunolocalization of amylase, $\mathrm{AQP} 5$, and $\mathrm{Na}^{+} / \mathrm{H}^{+}$exchangers (NHE1) was accomplished using a commercially available immunoperoxidase kit (Dako, Glostrup, Denmark). Formalin fixed, paraffin-embedded salivary gland tissues were sectioned to a thickness of $5 \mu \mathrm{m}$. The sections were deparaffinized and rehydrated by soaking the slides in xylene $(2 \times 5 \mathrm{~min})$, followed by an ethanol series $(100 \%, 95 \%$, and $70 \% ; 2 \times 3 \mathrm{~min}$ for each), and deionized water. To retrieve the antigens, the slides containing tissue sections were incubated in $1 \times$ Target Retrieval Solution, $\mathrm{pH} 6.0$ (Dako) at $125^{\circ} \mathrm{C}$ for $20 \mathrm{~min}$ in a decloaking chamber (Biocare Medical, Concord, CA, USA). The sections were cooled in ice water for $30 \mathrm{~min}$ and rinsed with deionized water for $5 \mathrm{~min}$. The endogenous peroxidase activity was blocked with phosphate buffer containing hydrogen peroxide, $15 \mathrm{mmol} / \mathrm{L} \mathrm{NaN}_{3}$, and detergent (peroxidase blocking solution, Dako) for $10 \mathrm{~min}$. The section was then dipped in 1X TBST buffer (Scy Tek Laboratories, West Logan, UT, USA) 2 times for 5 min each. The nonspecific protein binding background was blocked with $0.25 \%$ casein in serum-free protein block (Dako) for $10 \mathrm{~min}$ at room temperature. The primary antibodies for amylase $(1: 100$, Santa Cruz Biotechnology Inc., Dallas, TX, USA), AQP5 (1:100, Abcam, Cambridge, MA, USA) and NHE1 (1:100, Santa Cruz Biotechnology Inc.) were diluted in antibody diluent (Dako) and incubated at $4^{\circ} \mathrm{C}$ overnight. Then, the sections were rinsed in TBST buffer and treated with sec- 
ondary antibodies (anti-mouse, anti-rabbit, and anti-rabbit, respectively) for $1 \mathrm{~h}$ at room temperature. The sections were washed with TBST, and 3-amino-9-ethylcarbazole (AEC) substrate chromogen (Dako) was added. The sections were washed with deionized water and counter-stained with Harris hematoxylin (Sigma-Aldrich Co., St Louis, MO, USA). They were then washed thoroughly in tap water for $10 \mathrm{~min}$ and mounted with aqueous medium.

\section{Immunofluorescence}

Routinely processed paraffin-embedded SMG sections were deparaffinized and rehydrated. To enhance the immunoreactivity, the antigens were retrieved with $1 \times$ Target Retrieval Solution, pH 6.0 (Dako) using a decloaking chamber. Following a 10-min incubation in blocking solution (Dako), the tissues were incubated with mouse anti-amylase antibody (1:200; Santa Cruz Biotechnology Inc.) overnight at $4^{\circ} \mathrm{C}$. The tissue was then washed and stained with a fluorescein isothiocyanate (FITC)-conjugated anti-mouse secondary antibody (1:300; Sigma-Adrich Co.) for $1 \mathrm{~h}$. The sections were washed, blocked, and incubated with rabbit anti-AQP5 antibody (1:200, Abcam) overnight at $4^{\circ} \mathrm{C}$. The sections were then washed and incubated with a tetramethylrhodamine isothiocyanate (TRITC)-conjugated anti-rabbit secondary antibody (1:300) for $1 \mathrm{~h}$. They were then washed and mounted using vectashield mounting medium (with DAPI; Vector Laboratories, Burlingame, CA, USA). The fluorescence was examined using confocal microscopy (Olympus, Tokyo, Japan) using the FITC and TRITC channels.

\section{Intracellular $\mathrm{Ca}^{2+}$ measurement}

Human salivary gland (HSG) cells (ATCC) were cultured in Roswell Park Memorial Institute (RPMI)-1640 containing 5 mM glucose as described in. ${ }^{18}$ HSG cells were pre-incubated with or without BAPTA-AM and incubated with Fura-2 AM ( $2 \mu \mathrm{M}$, Molecular Probes; Eugene, OR, USA) for $30 \mathrm{~min}$ at $37^{\circ} \mathrm{C}$ in $\mathrm{KH}$ buffer $(145 \mathrm{mM} \mathrm{NaCl}, 5 \mathrm{mM} \mathrm{KCl}, 2.5 \mathrm{mM}$ $\mathrm{CaCl}_{2}, 1.2 \mathrm{mM} \mathrm{MgSO}$, $5 \mathrm{mM}$ glucose, $10 \mathrm{mM}$ HEPES, $\mathrm{pH}$ 7.4). After being loaded, cells were washed in $\mathrm{KH}$ buffer without $\mathrm{Ca}^{2+}$ and promptly treated with an IXD extract $(0.02 \mathrm{mg} / \mathrm{mL})$. Changes in the $\left[\mathrm{Ca}^{2+}\right]_{\mathrm{i}}$ were monitored by using an integrated spectrofluorometer (Photon Technology International, Birmingham, NJ, USA) with excitation ratios corresponding to 340 and $380 \mathrm{~nm}$ and emission at $512 \mathrm{~nm}$.

\section{Statistical analysis}

The results are presented as mean \pm standard error (SE). Group comparisons were performed using one-way analysis of variance, and Tukey's test was applied to the test and control conditions. $p<0.05$ was set as the criterion for statistical significance. Origin Pro version 9.0 was used for statistical calculations.

\section{Results}

\section{The effects of IXD extract on salivary parameters}

This study investigated the effects of type 1 diabetes mellitus on salivation and the beneficiary effects of an IXD extract on diabetes-induced dry mouth. A single spray of ethanolicextracted IXD was administered sublingually to normal or diabetic rats to observe the locally-acting effects of the drug. There was no significant difference in the saliva volume of vehicle- and IXD-treated control rats. A significant reduction in saliva output was observed in the vehicle-treated diabetic rats. However, saliva secretion was increased drastically in IXD-treated diabetic rats (Figure 2A). Similarly, the decreased salivary flow rate of the diabetic rats was improved significantly with IXD treatment (Figure 2B). The SMG weight was decreased significantly in the vehicle-treated
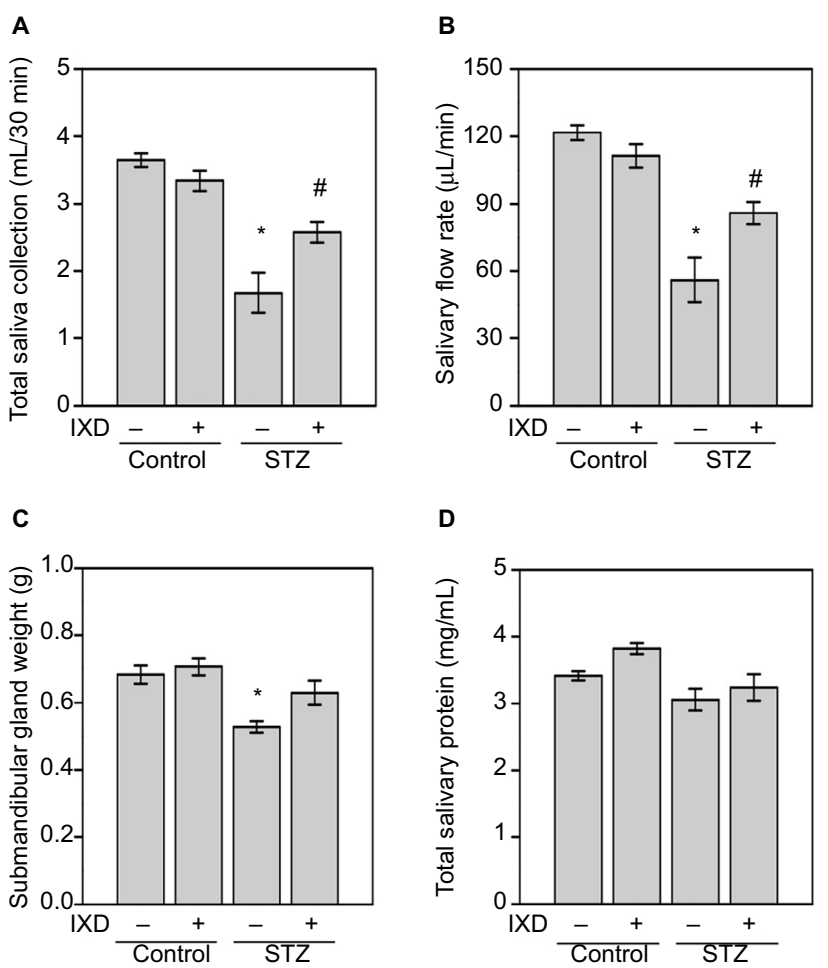

Figure 2 Effects of IXD extract on salivary parameters.

Notes: Saliva was collected for up to $30 \mathrm{~min}$ after pilocarpine injection and the salivary parameters were measured. (A) Total saliva collected in $30 \mathrm{~min}(\mathrm{~mL})$. (B) Salivary flow rate $(\mu \mathrm{L} / \mathrm{min})$. (C) Bilateral submandibular gland weight $(\mathrm{g})$. (D) Total salivary protein concentration $(\mathrm{mg} / \mathrm{mL})$. *significant difference vs vehicle-treated control rats ; " significant difference vs STZ-induced diabetic control rats $(p<0.05)$. Values are represented as mean \pm SEM ( $n=10$ rats per group).

Abbreviations: IXD, Ixeris dentata; SEM, standard error mean; STZ, streptozotocin. 
diabetic group, which might lead to the glandular dysfunction and hyposecretion; however, IXD extract increased salivary secretion with notable changes in SMG weight (Figure 2C). The total protein concentration remained unchanged in all the groups (Figure 2D). These results suggest that IXD extract increases saliva secretion and prevents hyposalivationinduced dry mouth.

\section{The effects of IXD extract on the SMG}

To investigate the effects of IXD extract in the morphology of the SMG, we performed a histological examination of salivary gland tissues stained with hematoxylin and eosin. Control rats, both with and without IXD treatment, had abundant acinar cells and well-preserved duct cells (Figure 3A, top). The
STZ-diabetic group, both with or without IXD treatment, had a depletion in the number of acinar cells and irregular ductal cell morphology in the SMG (Figure 3A, bottom). Both the sublingual route and spray form of the IXD treatment may have enhanced salivation without improving the morphology of the salivary gland.

Next, we performed CBB staining to ascertain the total protein expression in the saliva samples. We found that the protein expression was decreased in diabetic saliva, specifically a 55-kDa protein. The control rats treated with either water or IXD spray showed no differences in protein expression. The diabetic rats given IXD treatment had markedly increased expression of a protein at 55-kDa (Figure 3B). We then confirmed that the $55-\mathrm{kDa}$ protein was amylase

A

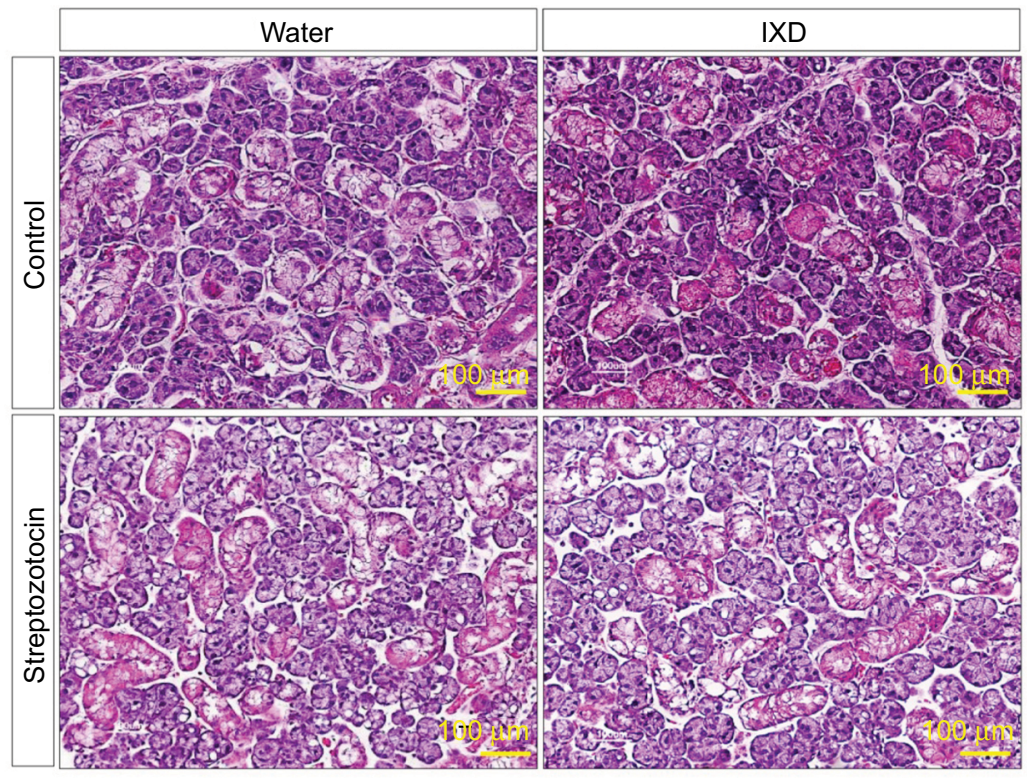

B

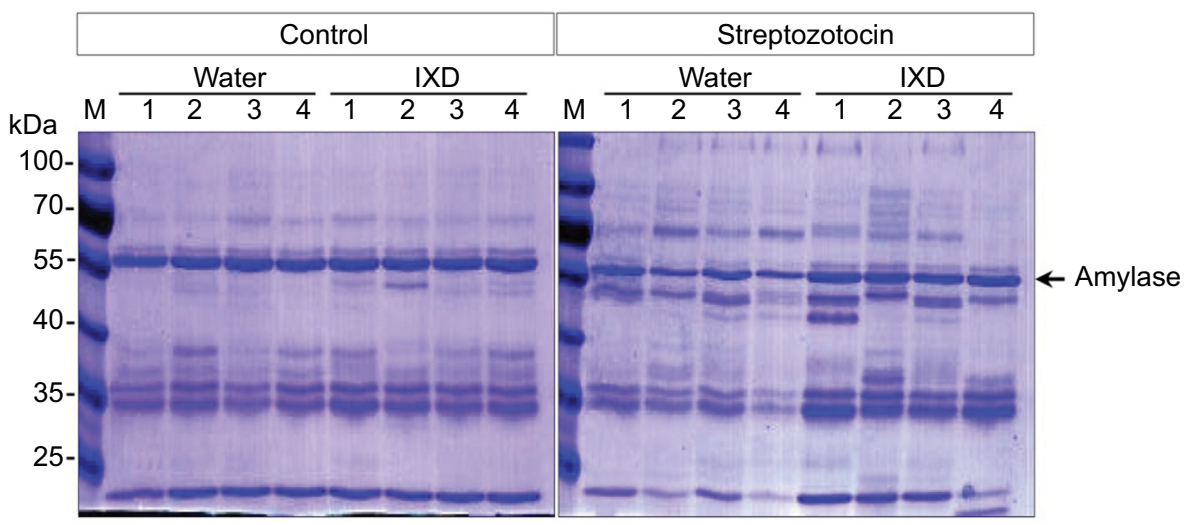

Figure 3 Effects of IXD extract on the morphology of submandibular glands and in salivary total protein expression.

Notes: (A) Hematoxylin and eosin staining was performed on paraffin-embedded submandibular gland tissues from normal and diabetic rats, either treated with water or IXD extract. Magnification=20×, scale bar=100 $\mu \mathrm{m}$. (B) CBB staining showing total protein expression in the saliva of control and diabetic rats, either treated with water or IXD extract. The same volume of saliva was loaded in each well of a 10\% SDS-PA gel, and the gel was stained with CBB. The black arrow indicates the molecular size of amylase $(55 \mathrm{kDa})$ present in the saliva.

Abbreviations: CBB, Coomassie Brilliant Blue; IXD, Ixeris dentata; SDS-PAGE, sodium dodecyl sulfate polyacrylamide gel electrophoresis. 
by Western blotting (Figure 4B). These results suggest that while a single spray of IXD extract did not improve the morphology of salivary gland cells (Figure 3A), it did induce a local response in saliva secretion (Figure 2A) and salivary $\alpha$-amylase expression (Figure 4 ). These results demonstrate that IXD extract has the ability to enhance salivary flow regardless of changes in glandular cell morphology.

\section{IXD extract increases salivary amylase expression in diabetic rats}

The expression of $\alpha$-amylase in saliva and salivary gland lysates was investigated using Western blot analysis. No significant differences were observed in control rats treated with either water or IXD extract. Vehicle-treated diabetic animals showed a significant reduction in amylase expression in both SMG tissue

A Submandibular gland

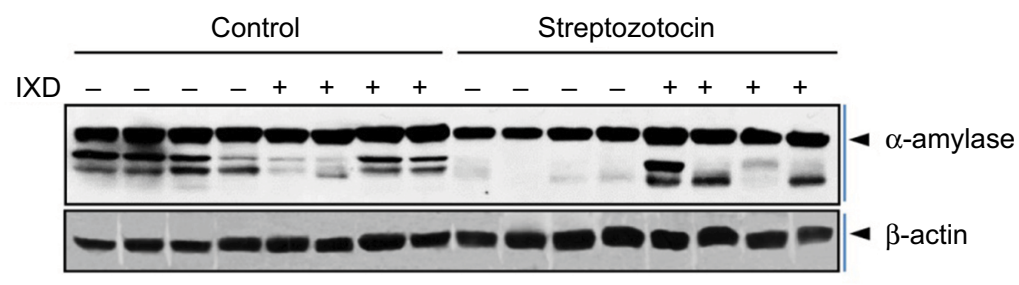

B

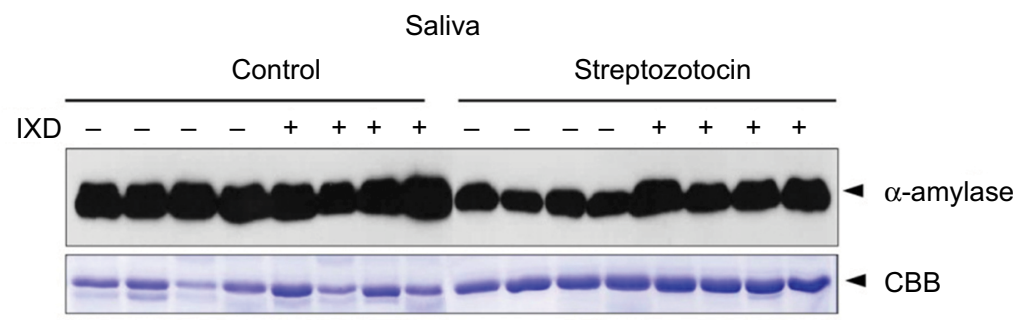

C $\alpha$-amylase

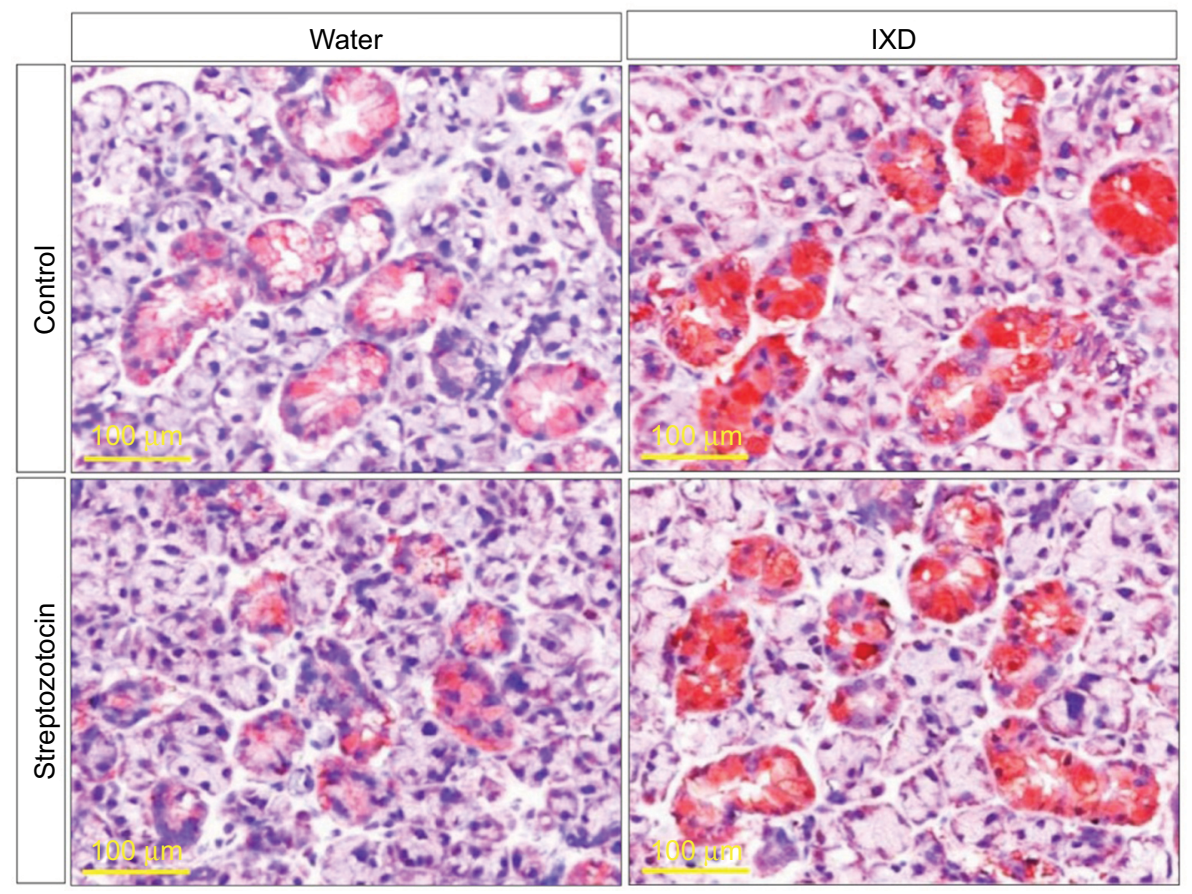

Figure $4 \alpha$-amylase protein expression was increased in IXD-treated diabetic rats

Notes: (A) Representative Western blot showing $\alpha$-amylase expression in submandibular gland tissue homogenates. (B) Western blot showing $\alpha$-amylase expression in saliva. (C) Immunohistochemical characterization showing the expression of $\alpha$-amylase in submandibular gland tissue. The red/brown color indicates positive expression of $\alpha$-amylase. Magnification $=40 \times$, scale bar $=100 \mu \mathrm{m}$.

Abbreviations: IXD, Ixeris dentata; CBB, Coomassie Brilliant Blue. 
homogenates (Figure 4A) and saliva (Figure 4B). Interestingly, the expression of amylase was increased almost 2-fold in IXD-treated diabetic rats in both tissue lysates (Figure 4A) and saliva (Figure 4B), which suggests that IXD extract can restore the decreased amylase folding and secretion seen in the salivary glands of diabetic animals.

We also ascertained the expression and localization of amylase in the SMGs of each test group. The immunohistochemistry data showed that amylase expression is abundant in both groups of healthy control rats (Figure 4C, top). The glands from the diabetic group showed less expression of $\alpha$-amylase in the acinar, ductal, and granular convoluted tubule cells (Figure 4C, bottom left); however, $\alpha$-amylase expression was restored in IXD-treated diabetic rats (Figure $4 \mathrm{C}$, bottom right). Previous studies suggest that the increase in salivary $\alpha$-amylase follows the increase of the salivary flow rate under non-stressful conditions. ${ }^{19}$ This implies a triggering of the acinar cells of the salivary glands for higher salivary $\alpha$-amylase production. Our data suggest that an increase in salivary secretion enhances salivary $\alpha$-amylase expression, which may play an important role in oral health.

\section{IXD extract increases salivary secretion through the activation of AQP5 channels}

Under normal physiological conditions, the presence of AQP5 in acinar cells aids in water movement to the lumen. ${ }^{20}$ In the present study, Western blot data showed that diabetic rats had significantly lower expression of AQP5 compared with control rats. A single spray of IXD extract increased AQP5 protein expression in diabetic SMG tissue homogenates (Figure 5A).

Multiple reports have shown the expression of AQP5 in various sites of the SMG. AQP5 is localized in the apical, basal, and lateral membranes of submandibular-gland acinar cells in SD rats, and the location is dependent on the level of AQP5 expression. ${ }^{20}$ Studies have also reported the expression of AQP5 in secretory acinar cells and in the intercalated duct cells of the SMG. ${ }^{21-23}$ Our study showed a uniform distribution of AQP5 expression in the control rats (Figure 5B, top); however, the diabetic rats had faint AQP5 expression in both the ductal and acinar cells of the SMG (Figure 5B, bottom). Further, we tested whether IXD extract could increase AQP5 expression. As expected, we observed strong and uniform

A Submandibular gland
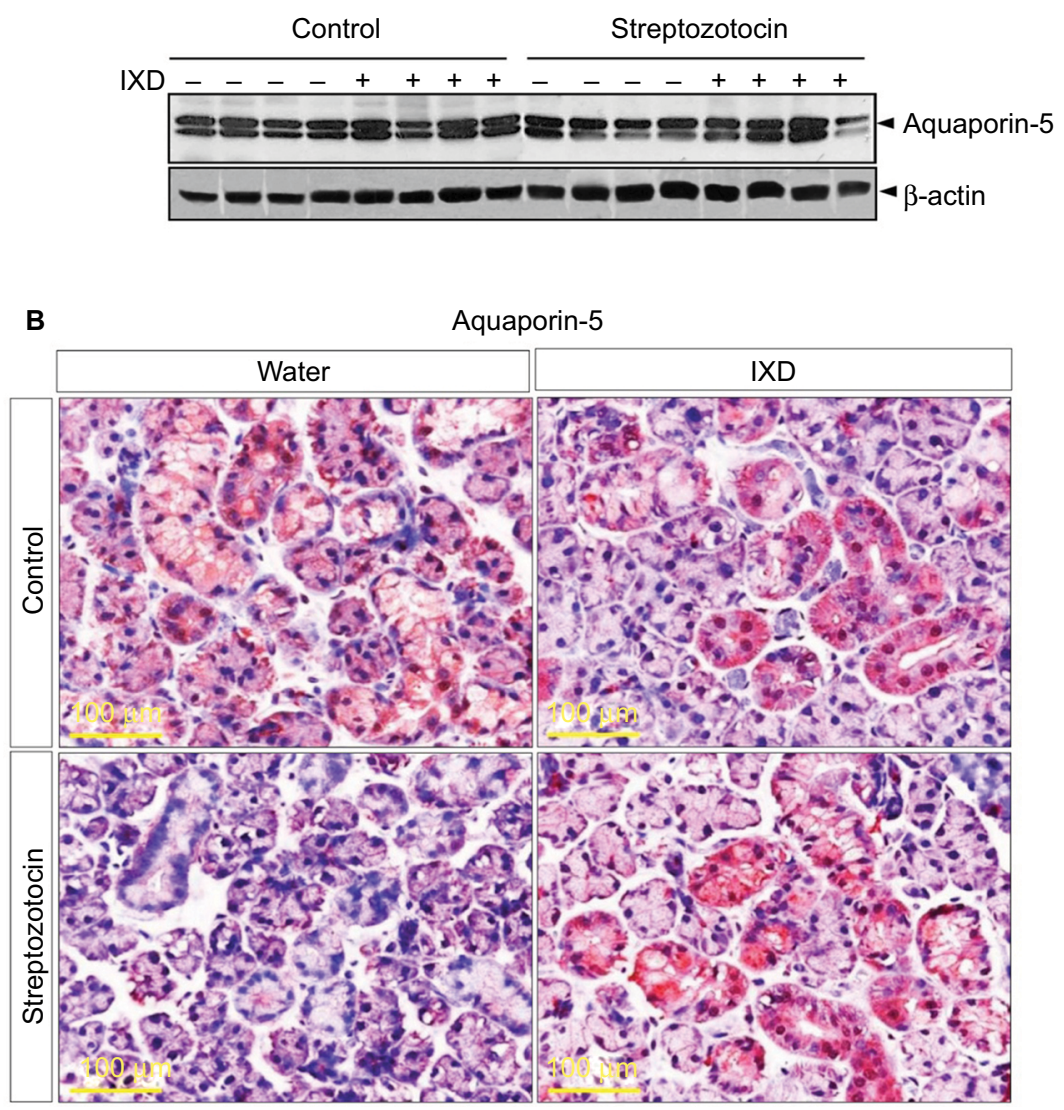

Figure 5 IXD extract increased AQP5 protein expression in submandibular gland tissue

Notes: (A) Representative Western blot showing AQP5 protein expression in SMG tissue homogenates. (B) Immunohistochemical analysis was performed on SMG tissue using anti-AQP5 antibody. The red/brown color indicates positive expression of AQP5. Magnification=40×, scale bar=100 $\mu \mathrm{m}$.

Abbreviations: AQP5, aquaporin-5; IXD, Ixeris dentata; SMG, submandibular gland. 
expression of AQP5 in both the salivary acinar and duct cells of diabetic rats treated with IXD (Figure 5B, bottom right). These data suggest that AQP5 regulates salivary secretion by increasing the membrane water permeability of acinar cells and is required for active isomolar fluid transport.

\section{Subcellular localization of $\alpha$-amylase and AQP5 in the SMG}

Double-labeled immunofluorescence for $\alpha$-amylase and AQP5 was performed on paraffinized rat SMG sections. The control rats, either treated or untreated with IXD extract, showed abundant amylase and AQP5 expression. Decreased amylase and AQP5 expression was observed in STZ-induced diabetic rats, but the expression was stronger in IXD-treated diabetic rats. The overlay images show that co-localization of amylase and AQP5 was high in the acinar cells and faint in the ductal cells of the SMG (Figure 6). Taken together, these data suggest that IXD extract treatment opens the AQP5 water

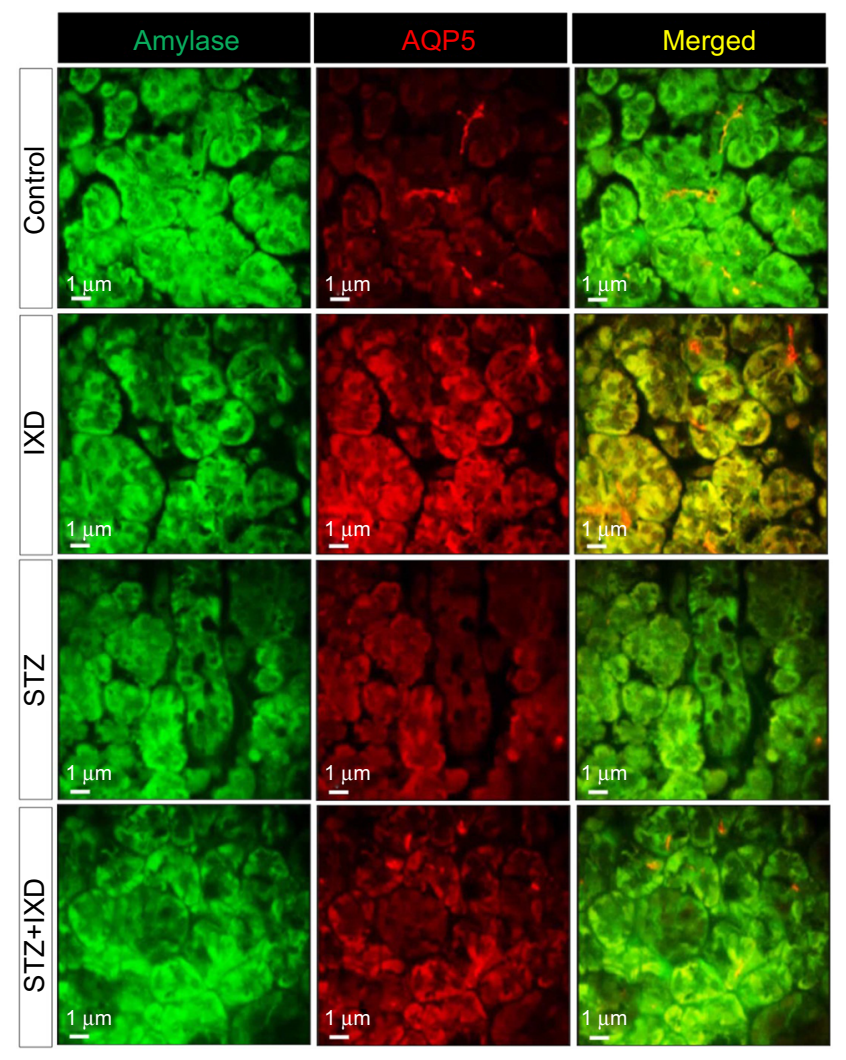

Figure 6 Confocal microscope images of immunofluorescence-detected $\alpha$-amylase and AQP5 expression

Notes: Vehicle-or STZ-injected rats were treated with either water or IXD extract to observe the expression and localization of amylase and AQP5 in the submandibular gland. Double immunofluorescence staining was done using antiamylase and anti-AQP5 antibodies. Green fluorescence indicates $\alpha$-amylase expression, red fluorescence indicates AQP5 expression, and yellow fluorescence indicates co-localization of both proteins. Treatment with IXD extract produced a high intensity of amylase and AQP5 fluorescence compared with the controls. Magnification $=40 \times$, scale bar $=1 \mu \mathrm{m}$.

Abbreviations: AQP5, aquaporin-5; STZ, streptozotocin; IXD, Ixeris dentata. channels and stimulates primary salivary secretion, offering a possible mechanism to treat xerostomia.

\section{High expression of NHEI increases salivary secretion in diabetic rats}

We found that NHE1 was highly expressed in ductal cells, and less so in acinar cells, in the control group. Furthermore, we observed weak expression of NHE1 in both the SMG duct and acinar cells of diabetic rats. As expected, IXD-treated rats showed higher expression of NHE1 in both the ductal and acinar cells of SMG tissue (Figure 7). Localization of NHE1 to acinar and duct cells suggests that $\mathrm{Na}^{+} / \mathrm{H}^{+}$antiporter isoform 1 might contribute to saliva secretion. These results suggest that IXD extract increases NHE1 expression, which may contribute to fluid secretion from acinar cells and absorption of $\mathrm{NaCl}$ by duct cells.

\section{IXD extract increases fluid secretion through the regulation of intracellular calcium}

The increase in cytosolic calcium is responsible for the saliva secretion. To determine the role of IXD extract in saliva secretion, we cultured HSG cells and promptly treated with IXD extract. Interestingly, we observed IXD-evoked $\left[\mathrm{Ca}^{2+}\right]_{\mathrm{i}}$ increase in the HSG cells (Figure 8A). Further, HSG cells were preincubated with BAPTA-AM, a high-affinity $\mathrm{Ca}^{2+}$ chelator that acts to block the $\mathrm{Ca}^{2+}$ in the cell. Pretreatment with BAPTA-AM for 20 min reduced the release of $\mathrm{Ca}^{2+}$ upon IXD treatment

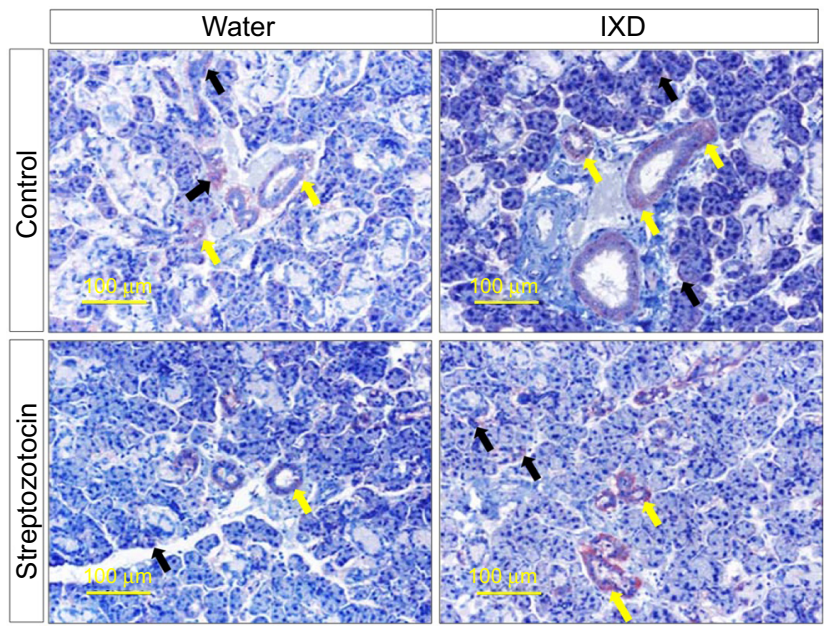

Figure 7 IXD extract treatment increased NHEI expression in rat submandibular glands

Notes: Representative immunohistochemical detection of NHEI in submandibular gland tissue from normal and diabetic rats, which were treated with either water or IXD extract. The yellow arrows indicate NHEI expression in duct cells and the black arrows indicate NHEI expression in acinar cells. Magnification=20×, scale bar $=100 \mu \mathrm{m}$.

Abbreviations: $\mathrm{NHEI}$, sodium/hydrogen exchanger-I; IXD, Ixeris dentata. 
(Figure 8B). These data suggest that IXD increases saliva secretion by increasing the release of calcium in HSG cells.

\section{Discussion}

Xerostomia is a serious concern for diabetic patients, elderly persons, and patients receiving radiation therapy for head and neck cancers. In our study, SMG weight was reduced significantly in diabetic rats, leading to a reduction in salivary secretion (Figure 2). The diminution in salivary flow leads to symptomatic drying of the oral tissues and loss of the protective effects of salivary buffers, proteins and mucins. ${ }^{24}$ There are various conflicting studies regarding salivary total protein levels and salivary amylase levels in diabetic patients, which have reported higher levels, ${ }^{25}$ lower levels of total protein, ${ }^{26}$ or no significant differences between diabetic and healthy non-diabetic patients. ${ }^{27}$ During our study,

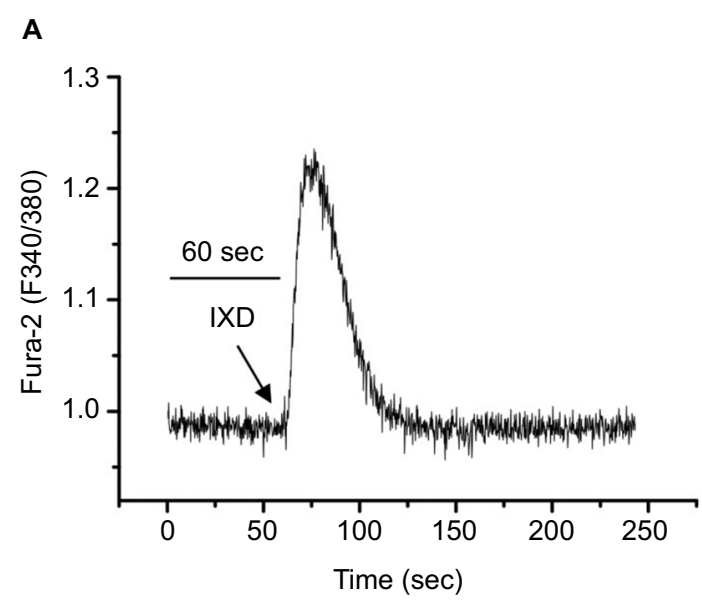

B

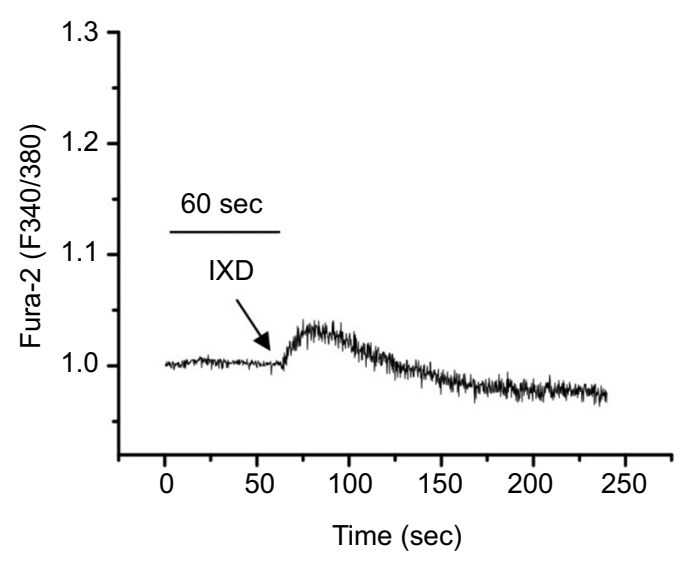

Figure 8 IXD extract increases intracellular calcium release in the HSG cells Notes: (A) Fura-2-loaded HSG cells were promptly treated with IXD extract $(0.02$ $\mathrm{mg} / \mathrm{mL}$ ) and changes in F340/F380 were monitored. (B) The effect of $10 \mu$ M BAPTAAM in IXD induced $\mathrm{Ca}^{2+}$ release. The figures represent the typical $\mathrm{Ca}^{2+}$ transients from more than three experiments.

Abbreviations: BAPTA-AM, I, 2-Bis (2-aminophenoxy) ethane- $N, N, N^{\prime}, N^{\prime}$ tetraacetic acid tetrakis (acetoxymethyl ester); $\mathrm{Ca}^{2+}$, calcium; HSG, human salivary gland; IXD, Ixeris dentata. no significant differences in total protein concentrations were observed among the control, IXD-treated control, and IXD-treated diabetic groups. However, the expression of $\alpha$-amylase was significantly reduced in the salivary glands of the diabetic group. Since salivary $\alpha$-amylase (1,4-alphaD-glucan glucanohydrolase; EC 3.2.1.1) is one of the most important enzymes in saliva ${ }^{28}$ and plays a critical role in the metabolism of complex carbohydrates, ${ }^{29}$ one could assume that salivary $\alpha$-amylase is useful in glucose homeostasis and also has a crucial role in secretory function. Our previous in vitro study showed that chronic exposure of HSG cells to a high concentration of glucose decreased amylase secretion. Furthermore, the purified IXD compound, as well as an IXD extract, increased amylase synthesis and secretion in HSG cells exposed to high levels of glucose. ${ }^{18}$ Therefore, for the in vivo studies, we treated diabetic rats with a sublingual spray of IXD extract and observed a significant increase in the salivary flow rate and $\alpha$-amylase expression in both glandular lysates and in the saliva (Figure 4). These results suggest that IXD extract regulates amylase folding and secretion and improves salivary gland cell function.

Aquaporins are proteins that form water-specific channels for water secretion. Among the various aquaporins (AQP1-AQP10), ${ }^{30}$ AQP5 is expressed in the apical, basal and lateral membranes ${ }^{21}$ of acinar cells in rat $\mathrm{SMGs}^{21,31}$ and is as an essential protein for salivary gland secretion. ${ }^{20}$ Hyposalivation is caused not only by changes in whole-body fluid homeostasis, but also by the reduction of membrane water-permeability from the loss of AQP5 in salivary gland acinar cells. ${ }^{16}$ Salivary acinar cells from AQP5-deficient mice have decreased membrane water-permeability and alterations in cell-volume regulation. ${ }^{10}$ In our study, IXD extract induced the expression of AQP5 in acinar and ductal cells of the salivary glands (Figure 5), which strongly suggests that IXD treatment opens water channels, leading to increased salivary secretion. Reduced AQP5 expression decreases water flow during the formation of primary saliva, and along with the increased salivary expression of sodium-dependent glucose transporter-1 (SGLT1), this may explain the reduced salivary flow rate in diabetic rats. ${ }^{16}$ Moreover, we performed double-labeled immunofluorescence for amylase and AQP5 and observed that both amylase and AQP5 were co-localized in SMG acinar cells, which are responsible for saliva secretion (Figure 6).

In this study, we have confirmed a 2-stage process of saliva secretion. IXD extract induced AQP5-mediated primary fluid secretion from acinar cells and also have shown the expression of NHE1, which have a role in the modifi- 
cation of primary saliva. Multiple studies suggest that the $\mathrm{Na}^{+} / \mathrm{H}^{+}$exchanger plays an important role in fluid secretion and $\mathrm{NaCl}$ absorption in salivary glands. ${ }^{32,33} \mathrm{NHE} 1$-deficient mice secrete significantly lower amounts of salivary fluid, ${ }^{32}$ suggesting the physiological role of NHE1 in the fluid secretion mechanism. NHE1 is localized in the basolateral membrane of ductal ${ }^{34}$ and acinar cells ${ }^{35}$ in rat SMGs. Consistent with these previous studies, we observed lower expression of NHE1, a sodium hydrogen antiporter-1, in both ductal and acinar cells of diabetic rats. IXD extract treatment induced the expression of NHE1 predominantly in the ductal region of the diabetic salivary glands (Figure 7). Park et al suggested that NHE1 induces salivation by regulating the flux of $\mathrm{Cl}^{-}$and $\mathrm{HCO}_{3}^{-}$ions across acinar cells to maintain the intracellular $\mathrm{pH}$ in both resting-state and muscarinic agonist-stimulated cells. ${ }^{32}$ These results indicate that the suppression of NHE1 expression severely restricts secretion, suggesting that this transport mechanism is meaningful for fluid secretion. The core mechanism of fluid secretion is the regulation of increase in $\left[\mathrm{Ca}^{2+}\right]_{\mathrm{i}}$. Our findings revealed that IXD extract treatment augmented intracellular calcium in HSG cells (Figure 8A) as well as AQP5 expression (Figure 5), thereby promoting salivary secretion. The increase in intracellular calcium in salivary gland cells regulates electrolytes and water channels that are demanded in the fluid secretion. ${ }^{36}$

\section{Conclusion}

Overall, our results suggest that patients with xerostomia can be treated with IXD extract to increase secretion of both fluid and proteins, specifically saliva and salivary amylase. We have reported that IXD extract is an effective sublingual treatment to directly activate the salivary gland for the regulation of salivary secretions. Our findings indicate that the conditions occurring in diabetes may be related to the consequence of alterations in the synthesis or folding of secretory proteins such as $\alpha$-amylase. Further studies should focus on the application of IXD extract in genetically modified animals.

\section{Acknowledgments}

This work was carried out with the support of the Cooperative Research Program for Agriculture, Science \& Technology Development (Project No. PJ01158104) funded by the Rural Development Administration of the Republic of Korea.

\section{Disclosure}

The authors report no conflicts of interest in this work.

\section{References}

1. Yi JM, Hong SH, Lee HJ, et al. Ixeris dentata green sap inhibits both compound 48/80-induced aanaphylaxis-like response and IgEmediated anaphylactic response in murine model. Biol Pharm Bull. 2002;25(1):5-9.

2. Lee MR, Lee HY, Lee GH, et al. Ixeris dentata decreases ER stress and hepatic lipid accumulation through regulation of ApoB secretion. Am J Chin Med. 2014;42(3):639-649.

3. Choi JS, Young HS, Kim BW. Hypoglycemic and hypolipemic effects oflxeris dentata in diabetic rats. Archiv Pharm Res. 1990;13(3):269-273.

4. Oh SH, Sung TH, Kim MR. Ixeris dentata extract maintains glutathione concentrations in mouse brain tissue under oxidative stress induced by kainic acid. J Med Food. 2003;6(4):353-358.

5. Sreebny LM, Yu A, Green A, Valdini A. Xerostomia in diabetes mellitus. Diabetes Care. 1992;15(7):900-904.

6. Nanci A. Ten Cate's Oral Histology: Development, Structure, and Function. Mosby Elsevier; 2014.

7. Pedersen AM, Bardow A, Jensen SB, Nauntofte B. Saliva and gastrointestinal functions of taste, mastication, swallowing and digestion. Oral Dis. 2002;8(3):117-129.

8. Villa A, Connell CL, Abati S. Diagnosis and management of xerostomia and hyposalivation. Ther Clin Risk Manage. 2015;11:45-51.

9. de Almeida Pdel V, Gregio AM, Machado MA, de Lima AA, Azevedo LR. Saliva composition and functions: a comprehensive review. J Contemp Dent Pract. 2008;9(3):72-80.

10. Krane CM, Melvin JE, Nguyen HV, et al. Salivary acinar cells from aquaporin 5-deficient mice have decreased membrane water permeability and altered cell volume regulation. J Biol Chem. 2001;276(26):23413-23420.

11. Steinfeld S, Cogan E, King LS, Agre P, Kiss R, Delporte C. Abnormal distribution of aquaporin-5 water channel protein in salivary glands from Sjogren's syndrome patients. Lab Invest. 2001;81(2):143-148.

12. Konttinen YT, Tensing EK, Laine M, Porola P, Tornwall J, Hukkanen M. Abnormal distribution of aquaporin- 5 in salivary glands in the NOD mouse model for Sjogren's syndrome. J Rheumatol. 2005;32(6):1071-1075.

13. Narang N, Sharma J. Sublingual mucosa as a route for systemic drug delivery. Int J Pharm Pharm Sci. 2011;3 (Suppl 2):18-22.

14. Thosar MM. Intra oral sprays -An overview. Int J Pharm Life Sci (IJPLS). 2011;2(11):1235-1246.

15. Deeds MC, Anderson JM, Armstrong AS, et al. Single dose streptozotocin-induced diabetes: considerations for study design in islet transplantation models. Lab Anim. 2011;45(3):131-140.

16. Romero AC, Ibuki FK, Nogueira FN. Sialic acid reduction in the saliva of streptozotocin induced diabetic rats. Arch Oral Biol. 2012;57(9):1189-1193.

17. Lee HY, Lee GH, Bhattarai KR, et al. Bax inhibitor-1 regulates hepatic lipid accumulation via ApoB secretion. Sci Rep. 2016;6:27799.

18. Lee HY, Lee GH, Kim HK, et al. Ixeris dentata-induced regulation of amylase synthesis and secretion in glucose-treated human salivary gland cells. Food Chem Toxicol. 2013;62:739-749.

19. Arhakis A, Karagiannis V, Kalfas S. Salivary alpha-amylase activity and salivary flow rate in young adults. Open Dent J. 2013;7:7-15.

20. Delporte C, Steinfeld S. Distribution and roles of aquaporins in salivary glands. Biochim Biophys Acta. 2006;1758(8):1061-1070.

21. Matsuzaki T, Suzuki T, Koyama H, Tanaka S, Takata K. Aquaporin-5 (AQP5), a water channel protein, in the rat salivary and lacrimal glands: immunolocalization and effect of secretory stimulation. Cell Tissue Res. 1999;295(3):513-521.

22. Nielsen S, King LS, Christensen BM, Agre P. Aquaporins in complex tissues. II. Subcellular distribution in respiratory and glandular tissues of rat. Am J Physiol. 1997;273(5 Pt 1):C1549-1561.

23. Gresz V, Kwon TH, Gong H, et al. Immunolocalization of AQP-5 in rat parotid and submandibular salivary glands after stimulation or inhibition of secretion in vivo. Am J Physiol Gastrointest Liver Physiol. 2004;287(1):G151-G161.

24. Fischer D, Ship JA. The effect of dehydration on parotid salivary gland function. Spec Care Dentist. 1997;17(2):58-64. 
25. Lopez ME, Colloca ME, Paez RG, Schallmach JN, Koss MA, Chervonagura A. Salivary characteristics of diabetic children. Braz Dent J. 2003;14(1):26-31.

26. Streckfus CF, Marcus S, Welsh S, Brown RS, Cherry-Peppers G, Brown RH. Parotid function and composition of parotid saliva among elderly edentulous African-American diabetics. J Oral Pathol Med. 1994;23(6):277-279.

27. Panchbhai AS, Degwekar SS, Bhowte RR. Estimation of salivary glucose, salivary amylase, salivary total protein and salivary flow rate in diabetics in India. J Oral Sci. 2010;52(3):359-368.

28. Zakowski JJ, Bruns DE. Biochemistry of human alpha amylase isoenzymes. Crit Rev Clin Lab Sci. 1985;21(4):283-322.

29. Mandel AL, Breslin PA. High endogenous salivary amylase activity is associated with improved glycemic homeostasis following starch ingestion in adults. $J$ Nutr. 2012;142(5):853-858.

30. Hosoi K. Physiological role of aquaporin 5 in salivary glands. Pflugers Arch. 2016;468(4):519-539.
31. Nishimura H, Yakeishi A, Saga T, Yamaki K. Effects of cevimeline on the immunolocalization of aquaporin-5 and the ultrastructure of salivary glands in Sjogren's syndrome model mice. Kurume Med J. 2009;56(3-4):39-47.

32. Park K, Evans RL, Watson GE, et al. Defective fluid secretion and $\mathrm{NaCl}$ absorption in the parotid glands of $\mathrm{Na}+\mathrm{H}+$ exchanger-deficient mice. J Biol Chem. 2001;276(29):27042-27050.

33. Catalan MA, Nakamoto T, Melvin JE. The salivary gland fluid secretion mechanism. J Med Invest. 2009;(56 Suppl):192-196.

34. Lee MG, Ohana E, Park HW, Yang D, Muallem S. Molecular mechanism of pancreatic and salivary gland fluid and $\mathrm{HCO} 3$ secretion. Physiol Rev. 2012;92(1):39-74.

35. Lee MG, Schultheis PJ, Yan M, et al. Membrane-limited expression and regulation of $\mathrm{Na}+\mathrm{H}+$ exchanger isoforms by $\mathrm{P} 2$ receptors in the rat submandibular gland duct. J Physiol. 1998;513(Pt 2):341-357.

36. Ambudkar IS. Polarization of calcium signaling and fluid secretion in salivary gland cells. Curr Med Chem. 2012;19(34):5774-5781.
Journal of Experimental Pharmacology

\section{Publish your work in this journal}

The Journal of Experimental Pharmacology is an international, peerreviewed, open access journal publishing original research, reports, reviews and commentaries on all areas of laboratory and experimental pharmacology. The manuscript management system is completely online and includes a very quick and fair peer-review system.

\section{Dovepress}

Visit http://www.dovepress.com/testimonials.php to read real quotes from published authors.

Submit your manuscript here: https://www.dovepress.com/journal-of-experimental-pharmacology-journal 